\title{
Vivencia de los cuidadores familiares de adultos mayores que sufren depresión
}

VIVÊNCIA DOS CUIDADORES FAMILIARES DE IDOSOS QUE SOFREM DEPRESSÃO

\author{
THE EXPERIENCE OF FAMILY CAREGIVERS OF OLDER PEOPLE WITH DEPRESSION
}

\author{
Luz Angélica Muñoz González¹, Yocelyn Margaret Price Romero², Marcia Reyes López³, \\ Macarena Ramírez ${ }^{4}$, Maguida Costa Stefanelli ${ }^{5}$
}

\section{RESUMEN}

La investigación tuvo como objetivo comprender cómo los cuidadores familiares vivencian la depresión de los adultos mayores, en su contexto cultural. Se realizó un estudio cualitativo de trayectoria etnográfica utilizando el referencial de Spradley. El universo cultural se constituyó con familiares, quienes ejercían el rol de cuidador, obteniéndose su participación a través de consentimiento informado. La enfermedad es percibida por los cuidadores como una limitación profunda en la calidad de vida del anciano, sumando soledad, tristeza y dolor. Los sentimientos de los pacientes deprimidos se hacen extensivos a los cuidadores, quienes también corren el riesgo de deprimirse. Las responsabilidades propias del cuidado también generan en los cuidadores sentimientos de culpa, lo cual los expone a un mayor grado de sufrimiento. Los familiares reconocen las formas de inicio de la enfermedad, sus atributos, las formas de tratamiento, y el estigma social asociado a la enfermedad mental y su rol fundamental de apoyo y cuidado en la recuperación.

\section{DESCRIPTORES}

Depresión.

Anciano.

Cuidadores.

Familia.

\section{RESUMO}

O objetivo da pesquisa foi compreender como os cuidadores familiares vivenciam a depressão dos idosos, no seu contexto cultural. Realizou-se um estudo qualitativo de trajetória etnográfica, utilizando-se o referencial de Spradley. O universo cultural constituiu-se de familiares que exerciam o papel de cuidador, obtendo-se sua participação através de consentimento informado. A doença é percebida pelos cuidadores como uma limitação profunda no seu modo de vida, com solidão, tristeza e dó. Os sentimentos dos pacientes deprimidos extendem-se aos cuidadores, e estes tem também o risco de tornarem-se deprimidos. As responsabilidades próprias do cuidado geram nos cuidadores sentimentos de culpa que produzem mais sofrimento. Os familiares reconhecem o início da doença, seus atributos, as formas de tratamento e o estigma social associado à doença mental, e seu papel fundamental de apoio e de cuidado no processo de recuperação.

\begin{abstract}
The objective of the study was to understand how family caregivers experience the depression of older people in their care, within their cultural context. This is a qualitative study of ethnographic trajectory, performed using Spradley as the framework. The cultural universe consisted of relatives who performed the role of caregivers, who provided informed consent. Caregivers perceived the disease as a deep limitation on their way of living, involving loneliness, sadness and pity. The feelings of the depressed patients extend to the caregiver, who also faces the risk of suffering from depression. The responsibilities of caring also generate feelings of guilt that produce more suffering. Relatives recognize the onset of the disease, their attributes, the forms of treatment, and the social stigma associated with this mental disease and that they have a fundamental role in providing support and care in the recovery process.
\end{abstract}

$\begin{array}{ll}\text { DESCRITORES } & \text { KEY WORDS } \\ \text { Depressão. } & \text { Depression. } \\ \text { Idoso. } & \text { Aged. } \\ \text { Cuidadores. } & \text { Caregivers. } \\ \text { Familia. } & \text { Family. }\end{array}$

\footnotetext{
${ }^{1}$ Enfermera. Doctor en Enfermería. Decana Facultad de Enfermería, Universidad Andrés Bello. Santiago, Chile. Imunoz@unab.cl ${ }^{2}$ Enfermera. Magíster en Enfermería. Candidato a Doctor en Enfermería, Servicio de Salud Metropolitano Sur Oriente. Santiago, Chile. yocelynp@gmail.com ${ }^{3}$ Licenciada en Enfermería, Universidad Andrés Bello. Santiago, Chile. marci.reyes@uandresbello.edu ${ }^{4}$ Licenciada en Enfermería Universidad Andrés Bello. Santiago, Chile. mac.ramirez@uandresbello.edu ${ }^{5}$ Enfermera. Profesor Titular y Doctor en Enfermería de la Escuela de Enfermería de Universidad de São Paulo. Profesor Visitante de Universidad Andrés Bello. São Paulo, SP, Brasil. mcstefanelli@terra.com.br
} 


\section{INTRODUCCIÓN}

Las enfermedades de salud mental son actualmente un gran problema de salud pública a nivel nacional e internacional, la cual ha requerido de la permanente implementación de políticas, planes y programas para buscar la mejor solución a ellos.

Las secuelas de las enfermedades de salud mental no se reducen sólo a la economía del país considerando los días de trabajo perdidos por licencias, disminución en la capacidad de ejecutar el trabajo, entre otros; sino que incluyen el impacto que éstas causan en la vida de la persona misma y de su entorno más cercano(1).

La depresión es una alteración patológica del estado de ánimo con descenso del humor que termina en tristeza acompañada de diversos síntomas y signos de tipo vegetativo, emocionales, del pensamiento, del comportamiento y de los ritmos vitales que persisten por tiempo habitualmente prolongado. Con frecuencia tiende a manifestarse en el curso de la vida, con aparición de varios episodios, adquiriendo un curso físico o recurrente con tendencia a la recuperación entre ellos ${ }^{(2)}$.

Es la enfermedad mental de mayor prevalencia a nivel mundial y se cree que será la segunda causa global de discapacidad en el año 2020. Las personas con depresión experimentan el doble de mortalidad que la población general y sufren mayores limitaciones en su funcionamiento psico-social que aquellas con enfermedades físicas crónicas como hipertensión y diabetes ${ }^{(3)}$.

La depresión es uno de los trastornos psiquiátricos más frecuentes del adulto mayor, diversos estudios epidemiológicos norteamericanos describen una prevalencia de depresión mayor de 1-5\% y una frecuencia de $8-27 \%$ de síntomas depresivos significativos en los adultos mayores que viven en la comunidad. La prevalencia es aún mayor en los sujetos mayores hospitalizados, y en aquellos residentes en instituciones de larga estadía(1).

En Chile, la depresión, es la segunda causa de años de vida saludable perdidos por discapacidad en muerte prematura (AVISA ${ }^{(a)}$ ) en mujeres. Esta patología es al menos dos veces más frecuente en mujeres que en hombres y tiene elevados costos directos e indirectos. Es responsable de un tercio de los días de trabajo perdidos debidos a enfermedad, concentrando más del $40 \%$ de las licencias médicas y representa al menos un $25 \%$ de las consultas de médicos generales en la Atención Primaria(4).

(a) Indicador compuesto que evalúa el número de años que una persona vive sin presentar sin ningún tipo de merma en su salud. Resulta de sumar los años perdidos por muerte prematura con los años vividos con discapacidad, que se restan de los años que podría vivir ese individuo en un tiempo y lugar determinados.
De los 20 trastornos específicos más relevantes por su contribución a los AVISA en Chile, para 2002, las depresiones mayores y los trastornos por el consumo de alcohol clasifican en primer y segundo lugar con $9,9 \%$ y $5,1 \%$, respectivamente. Los trastornos asociados al consumo de drogas y la esquizofrenia también se encuentran entre los 20 más importantes. El peso de los trastornos psiquiátricos específicos es incluso mayor cuando sólo se examinan aquellos que se presentan entre los 15 y 59 años de edad ${ }^{(2,4)}$.

La depresión es el trastorno afectivo o emocional más común en la vejez y suele responder a tratamientos, aun así, es importante considerar como esta enfermedad afecta la calidad de vida, incrementando el riesgo de suicidio, además se puede considerar un signo temprano de enfermedad crónica y resultado de enfermedad física, entendiendo entonces que las causas pueden ser tanto físicas como sicológicas. La deficiencia cognitiva puede deberse mas a la depresión que a la vejez o demencia. Cuando la depresión coexiste con enfermedades médicas, la falta de tratamiento demora la recuperación física. Los síntomas pueden ser secundarios a la interacción medicamentosa o a un cuadro físico no diagnosticado ${ }^{(3,5)}$.

Un adulto que entra en un periodo depresivo suele describir su ánimo como triste, sin esperanza, oscuro e inútil. La baja autoestima y la disminución de la energía física y psíquica llevan a un retraimiento apático con el entorno. Sin embargo existe otra dimensión de la depresión experimentada por un número significativo de adultos que expresan ansiedad, irritabilidad, agitación y sentimientos de angustia.

La prevalencia en la comunidad de depresión entre mayores de 65 años se ubica entre el 2 y $3 \%$ en Estados Unidos, en tanto la prevalencia de los últimos 6 meses en Santiago de Chile alcanza al 5,6\%, lo cual es más bajo que para la población general. Sin embargo, en instituciones tales como casas de reposo u hogares de ancianos, la prevalencia de depresión se eleva hasta un $17-20 \%$, con un 28 a $40 \%$ de los internos con síntomas depresivos $^{(4)}$

Por otra parte, según estudios de seguimiento, la presencia de depresión en pacientes geriátricos hospitalizados por causas no psiquiátricas sería un factor de riesgo para morir 30 meses después del diagnóstico ${ }^{(4)}$.

Otro agravante es el doble estigma que el adulto mayor con depresión lleva consigo. Uno, el de la enfermedad mental y el otro, relacionado a sus pérdidas biológicas. Con la cultura del cuerpo en occidente, el adulto mayor es considerado envejecido, estos factores contribuyen para la falta de independencia, su aislamiento, solidez y muchas veces para el desarrollo de la propia depresión o recaídas de éstas. Felizmente comienzan a surgir investigaciones en pro de una vejez exitosa, evidenciando ventajas que pueden ser estimuladas, principalmente en el plano afectivo ${ }^{(6)}$. 
Esto recalca la necesidad de reevaluar la función práctica de la enfermería en la atención del adulto mayor, la cual se ha basado tradicionalmente en el enfoque curativo. Es necesario dirigirla hacia el enfoque de promoción de la salud, considerando las áreas biológicas, sociales, psicológicas, culturales y espirituales como elementos de equilibrio necesario para alcanzar el bienestar.

La atención del adulto mayor es usualmente a largo plazo; se trata de brindarle una vida de calidad, en la que pueda utilizar al máximo sus capacidades físicas, psicológicas y sociales y hacer uso del apoyo que le brindan los familiares y amigos hasta el final de su vida.

Considerando que al conocer las vivencias por parte de los más cercanos a una persona depresiva y observando la forma en que la familia interactúa y se relaciona en torno a la enfermedad y su cotidianeidad, se puede ob- tener una contribución para el ejercicio de nuestro rol profesional.

\section{OBJETIVO}

El objetivo de este estudio fue comprender los significados que le atribuyen los cuidadores familiares de pacientes que sufren depresión en su contexto cultural.

\section{MÉTODO}

Se presenta una investigación cualitativa etnográfica ${ }^{(7)}$. El universo cultural de la investigación correspondió a 9 cuidadores familiares, cuyas edades fluctúan entre los 28 y 65 años y son de diversa condición socioeconómica y educacional (Cuadro 1).

Cuadro 1 - Universo Cultural de la Investigación - Santiago - 2008

\begin{tabular}{|c|c|c|c|c|c|}
\hline $\begin{array}{l}\text { Relación de } \\
\text { parentesco }\end{array}$ & Edad & Nivel Educacional & Ocupación & $\begin{array}{c}\text { Condición } \\
\text { Socioeconómica }\end{array}$ & Identificador \\
\hline Marido & 65 & Media Incompleta & Jubilado & Baja & E1 \\
\hline Sobrina & 28 & Básica Completa & Dueña de Casa & Media & E2 \\
\hline Esposa & 65 & Media Incompleta & Jubilada & Baja & E3 \\
\hline Hermana & 50 & Básica Incompleta & Dueña de casa & Media & E4 \\
\hline Sobrina & 28 & Media Completa & Dueña de casa & Media & E5 \\
\hline Sobrina & 25 & Técnico Profesional & Técnico Paramédico & Media & E6 \\
\hline Esposa & 65 & Básica incompleta & Dueña de casa & Baja & E7 \\
\hline Nuera & 40 & Técnico Profesional & Dueña de casa & Media & E8 \\
\hline Hija & 28 & Media incompleta & Dueña de casa & Media & E9 \\
\hline
\end{tabular}

Los criterios de inclusión para el estudio fueron: Familiares de pacientes con diagnóstico de depresión, que estuvieron en tratamiento por depresión en un centro asistencial de la ciudad de Santiago y que han estado en control al menos los últimos 6 meses, informantes con capacidad comunicativa, y finalmente quienes aceptaban el consentimiento informado. Los criterios de exclusión fueron familiar con depresión menor de 60 años y una red de apoyo insuficiente.

Se utilizaron como herramientas de recolección de datos: la observación participante, que fue realizada en tres etapas de realizar la observación en tres partes: observaciones descriptivas, las cuales deben ser más amplias y deben tratar de obtener una visión de la escena cultural, observación focalizada, luego del análisis de la observación anterior, y son mas estrechas, observación selectiva, las cuales tendrán por finalidad estrechar aún mas el campo de observación, la que nos permitió conocer las actividades de los familiares, las características físicas de la situación social en la que vine día a día y la satisfacción de participar en la escena cultural.
La entrevista en profundidad entendida como él medio por el cual se accederá a los contenidos de significado; es el evento único que ocurre entre dos personas: el informante y el entrevistador, estas tuvieron como finalidad comprender las perspectivas que tenían los cuidadores familiares, respecto de sus vidas y experiencias, buscando su visión de mundo, se realizaron en el domicilio de las familias. Todas las entrevistas fueron grabadas en su totalidad, siendo transcritas para mantener el discurso, respetando el sentido de lo expresado por los informantes ${ }^{(8)}$.

Para analizar los discursos se realizo en una primera etapa el análisis de Dominios que se refiere a una categoría de significado cultural que incluye otras categorías menores, este primer nivel de análisis se inicia a partir de las anotaciones de los discursos, de la cual se obtuvieron 21 Dominios Culturales, cabe destacar que la descripción de los dominios culturales envuelve siempre el uso del lenguaje, términos cubiertos, términos incluidos y las relaciones semánticas; son todas las palabras y frases que se defi- 
nen y dan significado a los objetos, acontecimientos y actividades observadas, así como los dominios identificados en una cultura, deben ser validados por los informantes, esto se realiza a través de preguntas estructuradas según los principios utilizados para formular este tipo de preguntas, cuyo objetivo es confirmar o desmentir dominios hipotéticos ${ }^{(7)}$.

A continuación se realizo el análisis taxonómico que es el segundo nivel de análisis de los datos, su objetivo es profundizar y demostrar la organización interna de un dominio. Así, como un dominio, una taxonomía revela subsectores de términos populares y la manera como están relacionados con un dominio como un todo, construyéndose finalmente 7 taxonomías; Describiendo la depresión, percibiendo el impacto de la depresión en la familia, cuidando a un familiar con depresión, la atención y tratamiento del familiar con depresión, el inicio y recidiva de la depresión, buscando soluciones, la relación entre el paciente y su familiar.

Y finalmente, se realizó el análisis de temas que corresponden a algún principio cognitivo, tácito o implícito, recurrente en varios dominios y que sirve como relación entre los subsistemas de significado cultural. De esta forma los temas culturales son grandes unidades de pensamiento, ellas consisten en un número de símbolos utilizados dentro de relaciones de significados.

\section{PRESENTANDO EL TEMA CULTURAL}

A partir del análisis de los datos para comprender el significado cultural en sus peculiaridades fue posible obtener la comprensión del significado de la depresión para los familiares cuidadores de personas con depresión. Vivenciando el impacto de la depresión es descrito a través de dos temas: Percibiendo la enfermedad y Cuidando al familiar con depresión.

\section{Vivenciando La Depresión de un miembro de la familia}

\section{Percibiendo la enfermedad}

La enfermedad es reconocida como algo complejo: esto haciendo referencia, principalmente a la forma de entregar cuidados, del sentimiento innato del familiar de querer dar apoyo, sin embargo percibiendo muchos obstáculos e impedimentos para otorgarlos. Esta percepción es considerada a partir de la falta de capacitación, con conocimientos suficientes para efectuar el cuidado de sus familiares.

El manejo de estos pacientes es muy complicado.

[...] uno con una enfermedad física, un dolor de cabeza, claro una aspirina y se la da y se calma un ratito, pero ya manejo la situación al menos por un par de horas, pero un enfermo de esa envergadura, no puedo uno, porque uno no está capacitado, si de repente se les escapan a los médicos, imagínese uno que no tiene ninguna capacidad, ningún estudio... entonces no es tan fácil [...] (E4).
Las formas de describir la enfermedad por los familiares la expresan a través de sus discursos como algo difícil de comprender y aceptar las diferentes formas de manifestación por personas distintas.

[...] una depresión ¿puede ser así?, ¿puede ser callado y no llorar? Porque a mi me da por llorar, me tiro a la cama y me desespero, y lloro y me da miedo y temores, entonces yo a eso pensaba, y como a él lo veía tan tímido, no llorando ni nada [...] y se puede haber manifestado de hace tiempo atrás? Y él no lo sabía [...] (E3)

La depresión la llamo yo porque es tristeza tan grande, ganas de dormir, ella no tenía ganas de nada (E2).

Los familiares destacan en los discursos de los pacientes, los cambios inexplicables de ánimo, el desinterés por la vida, y otras veces semejante a la pérdida de sentido de la vida. Dicen siempre la misma cosa, demostrando dificultad en la descripción de su sufrimiento. Así se percibe en el discurso la presencia de la idea del suicido.

\section{Una persona que está con enfermedad lo ve todo como un problema, no quiere saber de nada, no se levanta, no quie- re dormir, y lo único que quiere es morirse.}

Se angustian por no saber por qué se sienten tan mal, como si no tuvieran razón para vivir.

El mundo no me interesa, creo que muerta descansaría más, creo que llevo un tormento intolerable.

Es algo que nadie lo entiende porque es como si lo llevara marcado en su vida, es una enfermedad como un castigo que llevamos y lo llevaremos siempre (E7).

Los discursos siguientes sugieren las fases de alteración del ánimo entre depresión y euforia.

Cualquiera persona que la conozca, jamás pensará en su vida que es una persona depresiva, porque hay días que está alegre, sociable, pero nosotros sabemos que es un disfraz, porque la vemos encerrada sin deseos de levantarse.

[...] ella se siente sola a pesar de estar acompañada, se siente vieja y que no sirve para nada, pero dice que sí sirve (E1).

Estos discursos nos permiten identificar los sentimientos que afloran de los pacientes frente a su enfermedad a través de sus cuidadores, sus emociones relacionadas al fin del ciclo vital, pérdida de miembros de la familia, cambios fisiológicos, o simplemente por sentirse en la última etapa de la vida. Así, los cuidadores expresan las causas del inicio de la depresión.

El es así porque allá está solo, bueno la enfermedad de él empezó cuando se le murió la señora (E4).

Era una mujer feliz, alegre, hubo un cambio en mi vida cuando el hijo se fue al extranjero y le dio tanta pena porque se llevó a sus nietos, siempre estaba triste, con ganas de dormir y con unas ganas de llorar, ella no se conformó nunca. 
Ella tuvo problemas con su marido, fue muy raro porque siempre lo calló, siempre tenía pena, la escuchaba llorar en la noche, y no dormía (E8).

En estos discursos se refleja como los cuidadores identifican a la soledad como un factor predominante en el inicio de la depresión de su familiar. Es indiscutible que la soledad es una realidad para muchos adultos mayores; ya sea porque se encuentren efectivamente solos o simplemente se sientan solos, esto es un proceso muy doloroso, más aún cuando ésta se asocia a la pérdida del cónyuge, considerada como uno de los grandes conflictos en la vida de un ser humano.

El llegar a la etapa de adulto mayor, además implica un período en el que las metas y propósitos se modifican, se comienza a reevaluar los acontecimientos de la vida. En esta búsqueda se van encontrando dificultades de un pasado, que muchas veces, estaba protegido, oculto. El traer al presente aquellos recuerdos favorece estados depresivos.

Él tuvo muchos problemas cuando fue niño, tuvo problemas con el papá, entonces llegaba el papá, llegaba de trabajar y muy borracho, ¿comprende? [...] y él tenía que esconderse debajo de la cama dice, porque le pegaba a la mamá, le pegaba a ellos todo [...] si me dice yo tengo tantos problemas, por lo que nos hizo mi papá a nosotros $[\ldots](\mathrm{E} 3)$

Por otra parte, el universo cultural identifica la depresión como una enfermedad que pasa por etapas, ya que ven diversas formas de manifestación de ésta a lo largo de distintos períodos de tiempo en el actuar de su familiar:

[...] solamente a veces se siente decaída, como que no sé, como que no quiere hablar, pero eso no es todo el día, eso es un rato, después ella de repente se siente ella con ánimo, ella camina, habla, conversamos, vemos la televisión, entonces no es que ella este todo el día ahí [...] Ella decía: siento yo que no era parte de la casa, y no quería despertar y sentía todo el día sensación como si no durmiera en las noches, estuve hospitalizada y no me encontraron nada (E6).

Diferentes son las causas atribuibles a la depresión, pero la principal está también ligada con la etapa del ciclo vital en la que viven, en la cual son innumerables los cambios a los que se ve enfrentado, tanto en lo que se refiere a sí mismos, o bien en lo que se constituye como la dimensión social; como lo es el cambio de rol, esto; sumado a las pérdidas de sus seres más cercanos, hacen de éste un período complejo de la vida y si no se poseen herramientas adecuadas de adaptación o enfrentamiento pueden llevar a un trastorno depresivo.

En la enfermedad se viven distintas etapas, como sentirse extraña con su familia en su hogar, somnolencia a pesar de dormir bastante y luego más avanzada la enfermedad, somatización de la angustia.

Los cuidadores reconocen de forma muy clara los resultados o las consecuencias de tener depresión. Así los fami- liares determinan algunas características de la enfermedad que son atribuidas a la depresión.

No habla nada, él se encierra en su mundo, se enoja al tiro fuerte (E4).

De acuerdo a lo que socialmente es reconocido como un paciente con depresión es que los familiares le van asignando valor, sustento y significado a la enfermedad, sin saber que las manifestaciones de depresión son diferentes de acuerdo con la cultura, al tipo de personalidad, género y etapa del ciclo vital de la persona que padezca la enfermedad.

Es indudable que las experiencias de los familiares y el compartir la cotidianeidad de la vida con una persona que tiene depresión hacen que el cuidador o familiar más cercano vaya reconociendo e identificando la enfermedad de diferentes formas.

El universo cultural a lo largo de la investigación fue capaz de referirse a la depresión por su nombre, en la mayoría de los casos, pero en otros, los familiares se refieren a la enfermedad a través de la evolución que esta tiene o de acuerdo a alguna característica que manifiesta su familiar y la atribuyen a la depresión, dando la impresión de que no consideran o no perciben la persona del enfermo como tal.

Puros nervios, la cuestión que tiene, esta cosa (E9)

Si bien las familias conocen e identifican algunos aspectos de la enfermedad, de diversos modos se va expresando la negación como parte de la descripción, siendo un mecanismo de defensa, una forma de impedir que impacte al ser querido con depresión. Tal vez negando su existencia se protegen de sufrir, y de sumar dolor a su experiencia. El ver lo que está ocurriendo, es mucho más difícil, es enfrentar la vida y saber como permanecer en ella; tal vez es más simple vivir ciego de lo que ocurre alrededor, así hay una especie de protección, hay menos pesar.

Otro aspecto importante que los informantes manifiestan, se relaciona con la aparición de nuevos episodios depresivos, los que están dados por la falta de adhesividad al tratamiento medicamentoso. Este es uno de los principales problemas en la recuperación del paciente, el abandono del tratamiento suele llevar a los pacientes a recaídas ${ }^{(9)}$.

En éste ámbito cobra vital importancia el apoyo familiar, ya que los adultos mayores con depresión requieren de una sólida red de apoyo de sus seres más cercanos, no tan sólo para que lo ayuden en el seguimiento del tratamiento y los cuidados, sino que principalmente para sentir afecto, comprensión y apoyo por parte de quienes más quieren.

\section{Cuidando al familiar con depresión}

El tener una persona con depresión en el hogar, hace necesaria la participación familiar en el proceso de cuidarlo. Quienes toman este rol se ven con esta nueva responsa-
Vivencia de los cuidadores familiares de adultos mayores que sufren depresión González LAM, Romero YMP, López MR, Ramírez M, Stefanelli MC 
bilidad frente al cuidado de la persona. De alguna manera la familia se adapta, se reorganiza para atender a las actitudes que deben tener frente al paciente enfermo.

El encontrar familiares dispuestos a ayudarlos en estas circunstancias, en esta etapa es una tarea compleja, sobretodo considerando que los adultos mayores ya pudieran encontrarse solos por su etapa de vida. A menudo, hay solo una o dos personas que acompañan al paciente en su proceso de enfermedad, quedando fuera de participación el resto de la familia. El soporte económico y presencial de ellos en el tratamiento de la depresión es importante, pero hay que considerar que el tipo de cuidado requerido abarca diversas tareas que implican dedicación a diario, en lo cotidiano, así como el acompañamiento y otras muy propias de la depresión como el escuchar, observar y brindar apoyo cuando sea necesario.

La acompaño a todas partes, preparándole la comida, almorzando con ella, haciendo aseo, lo tira para arriba, lo saca del hoyo, le doy los remedios, escuchando sus atados estando alerta a sus cambios (E2).

Para ejercer este rol de cuidador se necesita dedicar tiempo para esta actividad, el que implica a menudo, redistribución de horarios, actividades cotidianas, espacio físico, incluso condiciones que influyen fuertemente en la vida del cuidador quien posterga ciertos aspectos de su vida cotidiana y de su propia salud por dedicarle tiempo al familiar depresivo ${ }^{(9)}$, llegando a generar sentimientos de culpa y obligación, ya que en muchas ocasiones se provoca un quiebre en su organización de vida y de familia.

Cada vez me cuesta salir más a trabajar, porque por ella me da un poco de cosa, dejé de participar en la Iglesia porque no quería dejar sola a mi mamá (E9).

Hay una gran alteración en toda la dinámica familiar, todos sufren con la depresión, parece que la enfermedad afecta la vida de todos (E7).

En el caso de aquellos que no viven junto a su familia, este hecho se hace más tangible aun, ya que además los costos económicos y en esfuerzos, por cuidar a su familiar se hacen mayores. La sensación de agobio, desesperación y estrés percibida por los familiares, son el factor común encontrado en el universo cultural estudiado. Los cuidadores reconocen que la enfermedad ha tenido repercusión en ellos, tanto físicas, como psicológicas. Cuando los informantes refieren sentimientos como me ha afectado harto(E5), me angustia(E8), me preocupa(E6), uno se desespera(E5).

Cuando reconocen el impacto de la depresión de su familiar en su propio bienestar parece existir una cierta comorbilidad familiar. Las expresiones vertidas por los informantes coinciden con el estudio(10), que señala que el cuidador domiciliario al mismo tiempo se percibe como alguien que cuida de otro, también percibe que para sí esto no es posible. Así sus necesidades físicas, emocionales y sociales, como dormir, descansar, trae un desgaste tal que compromete su salud.
Es agotador, me cuesta mucho a veces dormir (E7);

decaí en depresión, tenía mucho cargo en mi cabeza, me encuentro nerviosa, me despreocupo un poco de mi salud (E3).

Estas afirmaciones confirman que efectivamente la depresión impacta en la salud, especialmente en el plano emocional y psicológico, sintiendo el miedo de que la enfermedad se extienda a ellos.

Coopera para que el entorno se enferme (E6);

La enfermedad de ella me la está traspasando a mí (E1).

De este modo, la depresión se dimensiona como una enfermedad familiar, ello sugiere que la enfermedad no infecciosa, desde el paradigma biomédico, se percibe con riesgo de transmisión, en el ámbito de la teoría antropológica del contagio ${ }^{(11)}$. Para los cuidadores las posibilidades de contagio están relacionadas a las representaciones construidas sobre la depresión percibida como una enfermedad mental, física y sus consecuencias sobre las relaciones familiares, profesionales y sociales en que los pacientes se enfrentan en la cotidianeidad (11-12). Al abordar el tema de la enfermedad, la relaciona con el tema de la polución social, o sea el peligro, en este caso dado por la dimensión de la enfermedad que se instala y permanece por años haciendo sufrir(13).

Así, existe preocupación en el cuidador por sí mismo, y al simplemente escuchar sus discursos, es posible notar el cansancio y agotamiento psíquico que ellos también cargan día a día. Mientras están siendo agente de ayuda, también van transformándose en sujetos demandantes de cuidado.

Es importante destacar la preocupación por el resto de la familia, ya que muchas veces conviven con niños pequeños en el hogar, u otros adultos mayores, lo cual es causa de preocupación en los familiares, y se refleja en frases como:

Entonces como que uno el día no lo disfruta porque esta muy preocupada (E2).

Entonces para mi mama también es difícil, súper difícil (E9).

Entonces esto para mi mamá es una preocupación es una cruz que lleva ella, una carga. Mi hermana se las lloró todas (E9).

El impacto emocional evidenciado hasta acá puede ser reforzado con las frases a seguir que expresan lo que sufre el cuidador.

Uno tiene que estar por fuerza acá (E4).

Lo otro seria, desligarme, olvidarme y hacer así como que no existe y no puedo [...] (E8).

Tengo que seguir hasta el final no más [...] (E1).

No estoy en condiciones de cuidarlo, pero tampoco quiero dejarlo (E3). 
Uno quisiera ser como más fría y decir bueno, hasta aquí no mas, pero resulta que los sentimientos van por encima de muchas cosas [...] es complicado (E6).

Este deseo de muchas veces abandonar el rol de cuidador se complica por los sentimientos involucrados en la relación de estos familiares. Estas hacen pensar que el cuidador experimenta un sentimiento de obligación, de culpa frente al tener que asumir esta responsabilidad, que implica además de tener que modificar su propia vida para dedicar tiempo al cuidado, una carga psicológica y emocional que puede llevar al agotamiento total ${ }^{(13)}$.

Los sentimientos de angustia y sufrimiento se vuelven permanentes, reforzando el deseo de abandonar el rol. Las obligaciones para con el familiar, contiene esa dualidad de por una parte, no sentirse en las condiciones de seguir a cargo, y por otra con esa sensación de tener que permanecer junto a su familiar que se ha hecho dependiente de su atención, porque no queda otra alternativa. Así se vive en una ambivalencia.

Así, el sentimiento de culpa es intenso, como consecuencia no de haber cometido una mala acción como desatender algún aspecto del cuidado, sino por haber tenido la intención de abandonar el compromiso de amor y preocupación por el otro. En virtud de ello, se sienten sancionados por su conciencia y como sujeto histórico, tiene la condición de juzgar sus pensamientos y actos

El grupo de cuidadores señala que los lazos afectivos en la relación paciente-familia, es lo que los mantiene cumpliendo esta labor. El tema valórico sobre el bien y el mal, el amor y la conciencia moral exigen renuncias, que a pesar de lo adverso que pueda resultar cuidar a un paciente con depresión, motiva a éstos a continuar desempeñando su rol, aun cuando esto conlleva a anular su propio bienestar. Las familias muchas veces no ven soluciones reales ante la enfermedad de su familiar o simplemente prefieren no pensar en un futuro viviendo con la depresión.

No le vemos mucha salida a la situación, no me he puesto a pensar como va a ser el futuro (E9).

El universo cultural identifica, otras fuentes de tratamiento, más allá del psiquiátrico y farmacológico, que están dadas principalmente por la fe y la familia. Dios, Jesús,

\section{REFERÊNCIAS}

1. Hoyl T, Valenzuela E, Marín L, Pedro P. Depresión en el adulto mayor: evaluación preliminar de la efectividad, como instrumento de tamizaje, de la versión de 5 ítems de la Escala de Depresión Geriátrica. Rev Méed Chile. 2000;128(11):1199-204.

2. Chile. Ministerio de Salud. Tratamiento de personas con Depresión [texto na Internet]. Santiago; 2006 [citado 2008 jun. 7]. Disponível em: http://webhosting.redsalud.gov.cl/minsal/ archivos/guiasges/depresion.pdf la Biblia, toma un papel protagónico en esta búsqueda de soluciones, una forma de buscar respuestas a su enfermedad o como un modo de encontrar un soporte emocional en momentos difíciles.

Se metió en la iglesia evangélica, yo le leo párrafos de la Biblia, versículos y se queda tranquila (E1).

Se identifica a la familia como fuente importante de soporte en la búsqueda de soluciones, a pesar de que en esto hay ambivalencia, porque si bien saben que la familia es su principal sustento, y por tanto anhelan éste soporte, por otra parte predomina la sensación de ser un estorbo o peso, lo que en muchas ocasiones los lleva a no permitir ejercer su rol de cuidador.

Se hace evidente entonces la necesidad de recibir orientaciones y apoyo de los profesionales de la salud y de asociaciones dedicados al trastorno afectivo, principalmente a través de programas psico educacionales ${ }^{(14)}$ o por medio de otros formas de atención a la salud ${ }^{(15-16)}$, que posibilitan la percepción de que los cuidadores de adultos mayores, en general, no están solos en su experiencia. En lo caso de la depresión, los familiares, sean cuidadores o no, sufren como trastorno o como co-morbilidad familiar, lo que incluye tanto los cuidadores como la persona con depresión.

\section{CONSIDERACIONES FINALES}

El estudio permitió la comprensión de los significados de la vivencia de los cuidadores de pacientes adultos mayores que sufren depresión y que da cuenta de las repercusiones que el cuidado tiene para el familiar y para sí mismo, como responsable directo de la atención.

Así mismo los cuidadores perciben la enfermedad con desconocimiento, lo que les ocasiona profundas limitaciones en su modo de vida. Señalan que los sentimientos de los pacientes deprimidos se extienden a ellos mismos, por lo que tienen también el riesgo de deprimirse.

El estudio contribuirá al fortalecimiento de los programas educativos en cuanto a competencias de las enfermeras y equipo de salud, como también a la investigación y a los programas de salud familiar y comunitaria.

3. Organização Mundial da Saúde (OMS). Classificação de transtornos mentais e de comportamento: CID - 10. Critérios diagnósticos para pesquisa. Porto Alegre: Artes Médicas; 1998.

4. Vicente B, Kohn R, Saldivia S, Rioseco P. Carga del enfermar psíquico, barreras y brechas en la atención de Salud Mental en Chile. Rev Med Chile. 2007;135(12):1591-9.

5. Oliveira DAAP, Gomes L, Oliveira RF. Prevalência de depressão em idosos que freqüentam centros de convivência. Rev Saúde Pública. 2006;40(4):734-6.

Vivencia de los cuidadores familiares de adultos mayores que sufren depresión González LAM, Romero YMP, López MR, Ramírez M, Stefanelli MC 
6. Neri AL, Yassuda MS. Velhice bem-sucedida: aspectos afetivos e cognitivos. Campinas: Papirus; 2004.

7. Spradley J. The etnographic interview. New York: Holt Rinehart \& Winston; 1979.

8. Morse JM. Assuntos críticos en los métodos de investigación cualitativa. Colombia: Editorial Universidad de Antioquia; 2003.

9. Moreno RA, Stefanelli MC, Sousa MGG, Oliveira KC, Taveira ACA, Gentil V. Psychoeducational program for bipolar patients and their families. Bipolar Disord. 2005;7 Suppl 2:62.

10. Schossler T, Crossetti M. Cuidador domiciliar do idoso e o cuidado de si: uma análise através da teoria do cuidado humano de Jean Watson. Texto Contexto Enferm. 2008;17(2):280-7.

11. Douglas M. Risk and blame: essays in cultural theory. London: Routledge; 1992.
12. Muñoz LA. A doença veio para ficar: estudo etnográfico da vivencia do ser diabético [tese]. São Paulo: Escola de Enfermagem, Universidade de São Paulo; 1993.

13. Barroso SM, Bandeira ME, Nascimento E. Sobrecarga de familiares de pacientes psiquiátricos atendidos na rede pública. Rev Psiquiatr Clín. 2007;34(6):270-7.

14. Stefanelli MC, Moreno RA. Intervenção psicoeducacional: orientação e educação em saúde mental. In: Stefanelli MC, Fukuda IMK, Arantes EC, organizadores. Enfermagem psiquiátrica em suas dimensões assistenciais. Barueri: Manole; 2008. p. 281-95.

15. Diogo MJD'E, Ceolim, MF, Cintra FA. Orientações para idosas que cuidam de idosos no domicílio. Rev Esc Enferm USP. 2005;39(1):97-102.

16. Simonetti JP, Ferreira JC. Estratégias de coping desenvolvidas por cuidadores de idosos portadores de doença crônica. Rev Esc Enferm USP. 2008;42(1):19-25.

Proyecto financiado por DI 55-04-Universidad Andrés Bello (UNAB), Santiago, Chile. 